\title{
Cosmological particle creation in the little bang
}

\author{
S. V. Akkelin 1 \\ Bogolyubov Institute for Theoretical Physics, Metrolohichna 14b, 03143 Kyiv, Ukraine
}

(Received 2 September 2020; accepted 26 May 2021; published 22 June 2021)

\begin{abstract}
Particle production by expanding in the future light cone scalar quantum field is studied by assuming that the initial state is associated with the quasiequilibrium statistical operator corresponding to fluid dynamics. We calculate particle production from a longitudinally boost-invariant expanding quantum field designed as a simple but reliable model for the central rapidity region of a relativistic collision. Exact diagonalization of the model is performed by introducing a notion of quasiparticles.
\end{abstract}

DOI: $10.1103 /$ PhysRevD.103.116014

\section{INTRODUCTION}

It is noteworthy that relativistic hydrodynamics can be successfully applied for a phenomenological description of particle production in the relativistic nucleus and particle collisions; for a recent review see, e.g., Ref. [1]. Applicability of hydrodynamics for calculations of particle momentum spectra indicates the validity of some reduced descriptions for the state of a system. Such a reduced description can be fulfilled (and generalized hydrodynamic equations can be derived) utilizing Zubarev's formalism of a nonequilibrium statistical operator; see, e.g., Refs. [2-5] and also Refs. [6-10] for recent papers related to this method. A basic component of this formalism is the quasiequilibrium statistical operator. The quasiequilibrium statistical operator $\rho_{\sigma}^{q}$ is constructed by maximizing information entropy $[11,12]$ for the average local values of the energy-momentum density operator on a three-dimensional spacelike hypersurface $\sigma_{\mu}$ with a timelike normal vector $n_{\mu}(x)$ :

$$
n_{\mu}(x)\left\langle T_{\text {ren }}^{\mu \nu}(x)\right\rangle=n_{\mu}(x)\left\langle T_{\text {ren }}^{\mu \nu}(x)\right\rangle_{\sigma}^{q} .
$$

The left-hand side in the above equation is calculated with a true state of the system, and the right-hand side is calculated with the quasiequilibrium statistical operator, $\langle\ldots\rangle_{\sigma}^{q} \equiv \operatorname{Tr}\left[\rho_{\sigma}^{q} \ldots\right]$. Here $T_{\text {ren }}^{\mu \nu}(x)$ denotes the renormalized operator of an energy-momentum tensor: It is well known that expectation values of an energy-momentum tensor need regularization because expectation values of the products of quantum field operators taken in the same spacetime point are ill defined in the general case. The quasiequilibrium statistical operator reads

Published by the American Physical Society under the terms of the Creative Commons Attribution 4.0 International license. Further distribution of this work must maintain attribution to the author(s) and the published article's title, journal citation, and DOI. Funded by SCOAP ${ }^{3}$.

$$
\rho_{\sigma}^{q}=Z_{\sigma}^{-1} \exp \left(-\int_{\sigma} d \sigma n_{\mu}(x) \frac{u_{\nu}(x) T^{\mu \nu}(x)}{T(x)}\right)
$$

where $T(x)$ is the local temperature, $u_{\nu}(x)$ is hydrodynamical four-velocity, $u_{\nu}(x) u^{\nu}(x)=1, \sigma_{\mu}$ is a three-dimensional spacelike hypersurface with a timelike normal vector $n_{\mu}(x), T^{\mu \nu}(x)$ is the operator of an energy-momentum tensor, and $Z_{\sigma}$ is the normalization factor making $\operatorname{Tr}\left[\rho_{\sigma}^{q}\right]=1$. We assume for simplicity that a chemical potential $\mu=0$. Equation (1) defines the $u^{\nu}(x) / T(x)$ field in Eq. (2). It is worth noting that the quasiequilibrium statistical operator (2) can have a parametric time dependence even in the Heisenberg representation.

The zero temperature limit of such a statistical operator defines the corresponding ground state. If one has $n^{\mu}(x)=u^{\nu}(x)=(1, \mathbf{0})$, and $T=$ const, then $\int_{\sigma} d \sigma\left(n_{\mu}(x) u_{\nu}(x) T^{\mu \nu}(x) / T(x)\right)=H / T$ where $H$ is the Hamiltonian. In this case the ground state is $|0\rangle\langle 0|$, where $|0\rangle$ is the eigenstate of the Hamiltonian with the lowest eigenvalue and, therefore, coincides with the ordinary Minkowski vacuum. However, in general, $\int_{\sigma} d \sigma\left(n_{\mu}(x) u_{\nu}(x) T^{\mu \nu}(x) / T(x)\right) \neq H / T$, and therefore the ground state ("vacuum") of the quasiequilibrium statistical operator (2) may be different from the ordinary Minkowski vacuum. Perhaps the most straightforward definition of such a ground state can be performed by means of the instantaneous diagonalization of the operator $\int_{\sigma} d \sigma\left(n_{\mu}(x) u_{\nu}(x) T^{\mu \nu}(x) / T(x)\right)$ in some quasiparticle basis. The instantaneous ground state, $\left|0_{\sigma}\right\rangle$, can be defined as the eigenstate of this normal ordered operator with the lowest eigenvalue at the three-dimensional hypersurface $\sigma_{\mu}$. Of course, the existence of such an operator with a non-negative spectrum, bounded from below, is crucial to the existence and determination of the ground state containing no quasiparticle excitations.

The validity of the reduced description means that the quasiequilibrium statistical operator (2) can be utilized for 
(approximate) calculations of various operator-valued quantities, i.e., it can be regarded as a reliable proxy for a true state of a system. Typically, the expansion of an isolated system leads to a breakdown of the reduced description. Calculations at this far-from-equilibrium stage can be essentially simplified for the extreme case of a sudden decoupling of the quasiequilibrium state of a system which can be conditioned by switching off the interactions due to expansion. In the present paper we analyze particle creation after the sudden decoupling of a system utilizing simple but phenomenologically relevant model of the quasiequilibrium state for a boostinvariant expanding noninteracting boson quantum field. Unlike in previous studies [13-16], we perform exact diagonalization of the model in a quasiparticle basis. We show that the ground state of such a model is time dependent and does not coincide with the Minkowski vacuum. Then we estimate momentum spectra of particles produced as a result of the sudden decoupling of the quasiequilibrium state.

\section{DIAGONALIZATION OF THE QUASIEQUILIBRIUM STATISTICAL OPERATOR FOR BOOST-INVARIANT EXPANDING QUANTUM SCALAR FIELD}

In this section we diagonalize quasiequilibrium statistical operator (2) for the longitudinally boost-invariant Bjorken-type [17] expanding scalar field. This type of expansion is relevant for an ultrarelativistic collision and allows us to perform calculations analytically. Namely, we assume that matter produced in the little bang is locally restricted to the light cone beginning at the $t=z=0$ plane of the Minkowski spacetime manifold. Because the spacetime region occupied by the matter produced in an ultrarelativistic collision lies in the future light cone of a collision, it is convenient to introduce the Bjorken coordinates $(\tau, \eta)$ instead of the Cartesian ones $(t, z)$ :

$$
\begin{aligned}
& t=\tau \cosh \eta, \\
& z=\tau \sinh \eta,
\end{aligned}
$$

where $\eta$ is the so called spatial rapidity, $\tanh \eta=z / t$. The two other coordinates $\mathbf{r}_{T}=\left(r_{x}, r_{y}\right)$ are the Cartesian ones. One can see that $(\tau, \eta)$ coordinates cover the whole future light cone region. The Minkowski line element restricted to the light cone has then the form [we use the convention $\left.g^{\mu \nu}=\operatorname{diag}(+1,-1,-1,-1)\right]$ :

$$
d s^{2}=d t^{2}-d \mathbf{r}_{T}^{2}-d z^{2}=d \tau^{2}-d \mathbf{r}_{T}^{2}-\tau^{2} d \eta^{2} .
$$

Notice that $\tau$ is the proper time of observers which move with different but constant longitudinal velocities in such a way that their world lines begin at $z=t=0$. The corresponding longitudinally boost-invariant four-velocity $u^{\mu}$ is expressed as

$$
u^{\mu}(x)=(\cosh \eta, 0,0, \sinh \eta) .
$$

One can see that $\tau$ also controls a value of the four-velocity spacetime gradients,

$$
\partial_{\mu} u^{\mu}(x)=\frac{1}{\tau}
$$

We assume that a quasiequilibrium state is defined at a hypersurface with constant energy density in the comoving coordinate system for the field expanding with the fourvelocity (6). Then $T(x)$ is constant on the corresponding hypersurface, and such a hypersurface is defined by constant $\tau=\sqrt{t^{2}-z^{2}}$. This implies that

$$
\begin{gathered}
n^{\mu}(x)=u^{\mu}(x), \\
T(x)=T(\tau),
\end{gathered}
$$

and

$$
d \sigma=\tau d \eta d r_{x} d r_{y}
$$

Then the quasiequilibrium statistical operator (2) reads

$$
\rho_{\tau}^{q}=Z_{\tau}^{-1} \exp \left(-\frac{H^{[\tau]}}{T(\tau)}\right)
$$

where

$$
H^{[\tau]}=\int d \sigma u_{\mu}(x) u_{\nu}(x) T^{\mu \nu}(x)
$$

Here we are primarily interested in the effects of particle creation due to the expansion of quantum fields. Therefore we disregard field self-interactions and consider a scalar quantum field model with the classical action

$S=\int d t d^{3} r\left[\frac{1}{2}\left(\frac{\partial \phi}{\partial t}\right)^{2}-\frac{1}{2}\left(\frac{\partial \phi}{\partial \mathbf{r}}\right)^{2}-\frac{m^{2}}{2} \phi^{2}\right] \equiv \int d t d^{3} r L$.

Let us rewrite the classical action (13) in the Bjorken coordinates $(\tau, \eta)$. Taking into account that $d t d^{3} r=d \tau d \sigma$, we get

$$
S=\int d \tau d \sigma L
$$

where the Lagrangian density in such coordinates is

$L=\frac{1}{2}\left(\frac{\partial \phi}{\partial \tau}\right)^{2}-\frac{1}{2} \frac{1}{\tau^{2}}\left(\frac{\partial \phi}{\partial \eta}\right)^{2}-\frac{1}{2}\left(\frac{\partial \phi}{\partial \mathbf{r}_{T}}\right)^{2}-\frac{1}{2} m^{2} \phi^{2}$.

Taking into account that the energy-momentum tensor is 


$$
T^{\mu \nu}(x)=\partial^{\mu} \phi \partial^{\nu} \phi-g^{\mu \nu} L,
$$

it provides that the local energy density operator in the comoving frame, $u_{\mu} u_{\nu} T^{\mu \nu}(x)$, is

$$
\begin{aligned}
u_{\mu} u_{\nu} T^{\mu \nu}(x)= & \frac{1}{2}\left(\frac{\partial \phi}{\partial \tau}\right)^{2}+\frac{1}{2} \frac{1}{\tau^{2}}\left(\frac{\partial \phi}{\partial \eta}\right)^{2} \\
& +\frac{1}{2}\left(\frac{\partial \phi}{\partial \mathbf{r}_{T}}\right)^{2}+\frac{1}{2} m^{2} \phi^{2}
\end{aligned}
$$

where we take into account that $u_{\mu} \partial^{\mu}=\partial_{\tau}$.

It follows from Eq. (15) that conjugate momentum $\Pi^{[\tau]}$ with respect to $\tau$ is

$$
\Pi^{[\tau]}=\frac{\partial \phi(x)}{\partial \tau} .
$$

One can notice from Eqs. (12) and (17) that $H^{[\tau]}$ can be treated as an explicitly $\tau$-dependent "Hamiltonian" that generates translations in the timelike direction with respect to $\tau$. Evidently, such an operator does not coincide with the Hamiltonian $H$ that generates the translation with respect to $t$. Therefore, defining with respect to the $H^{[\tau]}$ instantaneous ground state $\left|0_{\tau}\right\rangle$, where $\tau$ is the instant to which it refers, does not coincide with the global vacuum state in flat Minkowski spacetime, $|0\rangle$, defined with respect to $H$.

In what follows we perform instantaneous diagonalization of $H^{[\tau]}$ in terms of some appropriate quasiparticle creation and annihilation operators. It allows us to distinguish contributions of the corresponding quasiparticles and the ground state (quasiparticle vacuum) to expectation values of relevant quantities. With this aim, it is necessary to find the representation of the canonical commutation relations at hypersurface $\tau=$ const,

$$
\left[\phi(x), \Pi^{[\tau]}\left(x^{\prime}\right)\right]=i \frac{1}{\tau} \delta\left(\eta-\eta^{\prime}\right) \delta^{(2)}\left(\mathbf{r}_{T}-\mathbf{r}_{T}^{\prime}\right)
$$

that diagonalizes $H^{[\tau]}$.

We start by noting that $\phi(x)$ obeys the Klein-Gordon equation,

$$
\left(\square-m^{2}\right) \phi(x)=0,
$$

where $\square=-\partial_{\mu} \partial^{\mu}$ is the d'Alembert operator associated with the Minkowski spacetime. It is well known that the solution of this equation in the future light cone can be written with the Hankel functions; see, e.g., Refs. [16,18-21]. Then

$$
\begin{aligned}
\phi(x)= & \int_{-\infty}^{+\infty} \frac{d^{2} p_{T} d \mu}{4 \pi \sqrt{2}}\left[-i e^{\mu \pi / 2+i \mu \eta+i \mathbf{p}_{T} \mathbf{r}_{T}} H_{i \mu}^{(2)}\left(m_{T} \tau\right) b\left(\mathbf{p}_{T}, \mu\right)\right. \\
& \left.+i e^{-\mu \pi / 2-i \mu \eta-i \mathbf{p}_{T} \mathbf{r}_{T}} H_{i \mu}^{(1)}\left(m_{T} \tau\right) b^{\dagger}\left(\mathbf{p}_{T}, \mu\right)\right]
\end{aligned}
$$

where $H_{i \mu}^{(1)}\left(m_{T} \tau\right)$ and $H_{i \mu}^{(2)}\left(m_{T} \tau\right)$ are the Hankel functions [22],

$$
\begin{array}{r}
H_{i \mu}^{(1)}\left(m_{T} \tau\right)=\frac{1}{i \pi} e^{\mu \pi / 2} \int_{-\infty}^{+\infty} d \vartheta e^{i m_{T} \tau \cosh \vartheta-i \mu \vartheta}, \\
H_{i \mu}^{(2)}\left(m_{T} \tau\right)=-\frac{1}{i \pi} e^{-\mu \pi / 2} \int_{-\infty}^{+\infty} d \vartheta e^{-i m_{T} \tau \cosh \vartheta+i \mu \vartheta}
\end{array}
$$

Here

$$
m_{T}=\sqrt{\mathbf{p}_{T}^{2}+m^{2}}
$$

is the so-called transverse mass and $\mathbf{p}_{T}=\left(p_{x}, p_{y}\right)$ is the transverse momentum. It is convenient to introduce notations

$$
\begin{gathered}
\tilde{H}_{i \mu}^{(1)}(x)=H_{i \mu}^{(1)}(x) e^{-\pi \mu / 2}, \\
\tilde{H}_{i \mu}^{(2)}(x)=H_{i \mu}^{(2)}(x) e^{\pi \mu / 2}
\end{gathered}
$$

Accounting for properties of the Hankel functions, one can see that

$$
\left[\tilde{H}_{i \mu}^{(1)}(x)\right]^{*}=\tilde{H}_{i \mu}^{(2)}(x) .
$$

Then, assuming

$$
\left[b\left(\mathbf{p}_{T}, \mu\right), b^{\dagger}\left(\mathbf{p}_{T}^{\prime}, \mu^{\prime}\right)\right]=\delta\left(\mu-\mu^{\prime}\right) \delta^{(2)}\left(\mathbf{p}_{T}-\mathbf{p}_{T}^{\prime}\right),
$$

with all other commutators vanishing, and by use of the identities (the Wronskian condition)

$$
\tilde{H}_{i \mu}^{(2) *}(x) \stackrel{\leftrightarrow}{\partial_{x}} \tilde{H}_{i \mu}^{(2)}(x)=-\frac{4 i}{\pi x},
$$

for the Hankel functions and their derivatives, one can see that representation (21) realizes the quantization procedure on the hypersurface $\tau=$ const; see Eqs. (18) and (19).

It is well known that the vacuum defined with respect to the Hankel functions coincides with the ordinary Minkowski vacuum defined with respect to the plane-wave modes, $b\left(\mathbf{p}_{T}, \mu\right)|0\rangle=0$ (see, e.g., Ref. [18]). To argue it, one can relate plane-wave modes with the Hankel functions. For this aim it is convenient to write the solution of the Klein-Gordon equation (20) with the plane-wave modes,

$\phi(x)=\int \frac{d^{3} p}{\sqrt{2 \omega_{p}}} \frac{1}{(2 \pi)^{3 / 2}}\left(e^{-i \omega_{p} t+i \mathbf{p r}} a(\mathbf{p})+e^{i \omega_{p} t-i \mathbf{p r}} a^{\dagger}(\mathbf{p})\right)$,

where 


$$
\omega_{p}=\sqrt{\mathbf{p}^{2}+m^{2}}
$$

The conjugated field momentum at the hypersurface $t=$ const is $\Pi=\frac{\partial \phi}{\partial t}$. The quantization prescription at such a hypersurface,

$$
\left[\phi(x), \Pi\left(x^{\prime}\right)\right]=i \delta^{(3)}\left(\mathbf{r}-\mathbf{r}^{\prime}\right)
$$

means that functions $a^{\dagger}(\mathbf{p})$ and $a(\mathbf{p})$ become creation and annihilation operators, respectively, which satisfy the following canonical commutation relations:

$$
\left[a(\mathbf{p}), a^{\dagger}\left(\mathbf{p}^{\prime}\right)\right]=\delta^{(3)}\left(\mathbf{p}-\mathbf{p}^{\prime}\right),
$$

and $\left[a(\mathbf{p}), a\left(\mathbf{p}^{\prime}\right),\right]=\left[a^{\dagger}(\mathbf{p}), a^{\dagger}\left(\mathbf{p}^{\prime}\right)\right]=0$. Then, comparing Eq. (21) with Eq. (30) and using Eqs. (22) and (23), one can easily get (see, e.g., Ref. [16]) that

$$
\begin{aligned}
a(\mathbf{p}) & =\frac{1}{\sqrt{2 \pi \omega_{p}}} \int_{-\infty}^{+\infty} d \mu e^{i \mu \theta} b\left(\mathbf{p}_{T}, \mu\right), \\
a^{\dagger}(\mathbf{p}) & =\frac{1}{\sqrt{2 \pi \omega_{p}}} \int_{-\infty}^{+\infty} d \mu e^{-i \mu \theta} b^{\dagger}\left(\mathbf{p}_{T}, \mu\right) .
\end{aligned}
$$

Here $\theta$ is momentum rapidity and $\tanh \theta=p_{z} / \omega_{p}, p_{z}$ is the longitudinal momentum. Taking into account Eq. (31) one can write

$$
\begin{gathered}
\omega_{p}=m_{T} \cosh \theta, \\
p_{z}=m_{T} \sinh \theta,
\end{gathered}
$$

where transverse mass $m_{T}$ is defined by Eq. (24). It follows from Eqs. (34) and (35) that " $a$ " and " $b$ " particles are defined with respect to the same vacuum.

Substituting (21) into Eq. (17), and performing integrations over spacetime variables in Eq. (12), we bring $H^{[\tau]}$ to the form

$$
\begin{aligned}
H^{[\tau]}= & \frac{1}{2} \int_{-\infty}^{+\infty} d^{2} p_{T} d \mu \omega\left(p_{T}, \mu, \tau\right)\left[E\left(p_{T}, \mu, \tau\right)\left(b^{\dagger}\left(\mathbf{p}_{T}, \mu\right) b\left(\mathbf{p}_{T}, \mu\right)+b\left(\mathbf{p}_{T}, \mu\right) b^{\dagger}\left(\mathbf{p}_{T}, \mu\right)\right)\right. \\
& \left.-F\left(p_{T}, \mu, \tau\right) b\left(\mathbf{p}_{T}, \mu\right) b\left(-\mathbf{p}_{T},-\mu\right)-F^{*}\left(p_{T}, \mu, \tau\right) b^{\dagger}\left(\mathbf{p}_{T}, \mu\right) b^{\dagger}\left(-\mathbf{p}_{T},-\mu\right)\right]
\end{aligned}
$$

where

$$
\omega\left(p_{T}, \mu, \tau\right)=\sqrt{m_{T}^{2}+\frac{\mu^{2}}{\tau^{2}}},
$$

and we introduced notations

$$
\begin{aligned}
E\left(p_{T}, \mu, \tau\right) & =\frac{\pi \tau}{4 \omega\left(p_{T}, \mu, \tau\right)}\left[\left|\partial_{\tau} \tilde{H}_{i \mu}^{(2)}\left(m_{T} \tau\right)\right|^{2}+\omega^{2}\left(p_{T}, \mu, \tau\right)\left|\tilde{H}_{i \mu}^{(2)}\left(m_{T} \tau\right)\right|^{2}\right] \\
F\left(p_{T}, \mu, \tau\right) & =\frac{\pi \tau}{4 \omega\left(p_{T}, \mu, \tau\right)}\left[\left(\partial_{\tau} \tilde{H}_{i \mu}^{(2)}\left(m_{T} \tau\right)\right)^{2}+\omega^{2}\left(p_{T}, \mu, \tau\right)\left(\tilde{H}_{i \mu}^{(2)}\left(m_{T} \tau\right)\right)^{2}\right]
\end{aligned}
$$

Using Eq. (29) one can get that

$$
E^{2}\left(p_{T}, \mu, \tau\right)-\left|F\left(p_{T}, \mu, \tau\right)\right|^{2}=1
$$

As one sees from Eq. (38), $H^{[\tau]}$ is nondiagonal in the creation and annihilation operators. Diagonalization of $H^{[\tau]}$ can be performed by means of a canonical Bogolyubov transformation. ${ }^{1}$ The corresponding quasiparticle creation, $\xi^{\dagger}$, and destruction, $\xi$, operators with canonical commutation relations

$$
\left[\xi\left(\mathbf{p}_{T}, \mu, \tau\right), \xi^{\dagger}\left(\mathbf{p}_{T}^{\prime}, \mu^{\prime}, \tau\right)\right]=\delta\left(\mu-\mu^{\prime}\right) \delta^{(2)}\left(\mathbf{p}_{T}-\mathbf{p}_{T}^{\prime}\right)
$$

\footnotetext{
${ }^{1}$ Our treatment is similar to the one required to diagonalize a Hamiltonian in an expanding curved spacetime; see, e.g., Ref. [23] and references therein.
} 
and $\left[\xi^{\dagger}\left(\mathbf{p}_{T}, \mu, \tau\right), \xi^{\dagger}\left(\mathbf{p}_{T}^{\prime}, \mu^{\prime}, \tau\right)\right]=0,\left[\xi\left(\mathbf{p}_{T}, \mu, \tau\right), \xi\left(\mathbf{p}_{T}^{\prime}, \mu^{\prime}, \tau\right)\right]=0$, are related to $b^{\dagger}$ and $b$ operators through a Bogolyubov transformation with $\tau$-dependent coefficients $\alpha\left(\mathbf{p}_{T}, \mu, \tau\right)$ and $\beta\left(\mathbf{p}_{T}, \mu, \tau\right)$ :

$$
\begin{gathered}
b\left(\mathbf{p}_{T}, \mu\right)=\alpha\left(\mathbf{p}_{T}, \mu, \tau\right) \xi\left(\mathbf{p}_{T}, \mu, \tau\right)+\beta^{*}\left(\mathbf{p}_{T}, \mu, \tau\right) \xi^{\dagger}\left(-\mathbf{p}_{T},-\mu, \tau\right), \\
b^{\dagger}\left(\mathbf{p}_{T}, \mu\right)=\alpha^{*}\left(\mathbf{p}_{T}, \mu, \tau\right) \xi^{\dagger}\left(\mathbf{p}_{T}, \mu, \tau\right)+\beta\left(\mathbf{p}_{T}, \mu, \tau\right) \xi\left(-\mathbf{p}_{T},-\mu, \tau\right), \\
\left|\alpha\left(\mathbf{p}_{T}, \mu, \tau\right)\right|^{2}-\left|\beta\left(\mathbf{p}_{T}, \mu, \tau\right)\right|^{2}=1 .
\end{gathered}
$$

It follows from Eqs. (44), (45), and (46) that

$$
\begin{aligned}
& \xi\left(\mathbf{p}_{T}, \mu, \tau\right)=\alpha^{*}\left(\mathbf{p}_{T}, \mu, \tau\right) b\left(\mathbf{p}_{T}, \mu\right)-\beta^{*}\left(\mathbf{p}_{T}, \mu, \tau\right) b^{\dagger}\left(-\mathbf{p}_{T},-\mu\right), \\
& \xi^{\dagger}\left(\mathbf{p}_{T}, \mu, \tau\right)=\alpha\left(\mathbf{p}_{T}, \mu, \tau\right) b^{\dagger}\left(\mathbf{p}_{T}, \mu\right)-\beta\left(\mathbf{p}_{T}, \mu, \tau\right) b\left(-\mathbf{p}_{T},-\mu\right) .
\end{aligned}
$$

Substituting Eqs. (44) and (45) into Eq. (38) and requiring diagonalization of $H^{[\tau]}$ in operators $\xi^{\dagger}$ and $\xi$ we obtain

$$
2 E \alpha \beta-F \alpha^{2}-F^{*} \beta^{2}=0
$$

One can see from the above equation that $\beta / \alpha$ is a solution of the quadratic equation. Then, using Eq. (42) we get $\frac{\beta}{\alpha}=\frac{E \pm 1}{F^{*}}$. Choosing the solution which tends to zero when $\tau$ tends to infinity, we get

$$
\frac{\beta}{\alpha}=\frac{E-1}{F^{*}} .
$$

This implies that

$$
\frac{|\beta|^{2}}{1+|\beta|^{2}}=\frac{(E-1)^{2}}{|F|^{2}}
$$

where we used Eq. (46). Taking into account (42) we finally get

$$
|\beta|^{2}=\frac{E-1}{2} .
$$

Also, substituting Eq. (52) into Eq. (50) we get

$$
\alpha \beta^{*}=\frac{1}{2} F^{*} .
$$

It is noteworthy that Eqs. (52) and (53) allow us to rewrite Eqs. (40) and (41) in the form

$$
\begin{aligned}
\tilde{H}_{i \mu}^{(2)}\left(m_{T} \tau\right)= & \left(\frac{2}{\pi \tau \omega\left(p_{T}, \mu, \tau\right)}\right)^{1 / 2} \\
& \times\left(\alpha^{*}\left(\mathbf{p}_{T}, \mu, \tau\right)+\beta\left(\mathbf{p}_{T}, \mu, \tau\right)\right),
\end{aligned}
$$

$$
\begin{aligned}
\partial_{\tau} \tilde{H}_{i \mu}^{(2)}\left(m_{T} \tau\right)= & -i\left(\frac{2 \omega\left(p_{T}, \mu, \tau\right)}{\pi \tau}\right)^{1 / 2} \\
& \times\left(\alpha^{*}\left(\mathbf{p}_{T}, \mu, \tau\right)-\beta\left(\mathbf{p}_{T}, \mu, \tau\right)\right) .
\end{aligned}
$$

Expressions for $\tilde{H}_{i \mu}^{(1)}\left(m_{T} \tau\right)$ and $\partial_{\tau} \tilde{H}_{i \mu}^{(1)}\left(m_{T} \tau\right)$ follow from Eq. (27) and Eqs. (54) and (55).

Under this transformation $H^{[\tau]}$ takes the form

$$
H^{[\tau]}=\int_{-\infty}^{+\infty} d^{2} p_{T} d \mu \omega\left(p_{T}, \mu, \tau\right) \xi^{\dagger}\left(\mathbf{p}_{T}, \mu, \tau\right) \xi\left(\mathbf{p}_{T}, \mu, \tau\right),
$$

where we omitted a constant term taking into account that such a term is canceled in the expression (11) for the quasiequilibrium statistical operator. One can see that $\omega\left(p_{T}, \mu, \tau\right)$ has the meaning of the energy of the quasiparticle. A direct consequence of the Bogolyubov transformation (44), (45), and (46) is that the notion of a vacuum is not unique for " $b$ " and " $\xi$ " particles. Namely, the ground state of the "Hamiltonian" $H^{[\tau]}$ is a $\tau$-dependent highly entangled squeezed state (see, e.g., Refs. [24,25]) of correlated pairs of $b^{\dagger}\left(\mathbf{p}_{T}, \mu\right)$ and $b^{\dagger}\left(-\mathbf{p}_{T},-\mu\right)$ quanta with zero total momentum. One can draw an analogy between the absence of a unique ground ("vacuum") state in the Little Bang created in a relativistic collision, and absence of a unique vacuum state in the Big Bang cosmological expansion (see, e.g., Refs. [18,23]).

It is now a simple matter to write expectation values of $\xi$ and $\xi^{\dagger}$ quasiparticle operators with the quasiequilibrium statistical operator $(11),\langle\ldots\rangle_{\tau}^{q} \equiv \operatorname{Tr}\left[\rho_{\tau}^{q} \ldots\right]$. Because $H^{[\tau]}$ has the thermal-like diagonal form in the quasiparticle representation [see Eq. (56)] it can be done utilizing the 
thermal Wick theorem [26] (see also Refs. [27,28] and Ref. [16]). We then obtain

$$
\left\langle\xi^{\dagger} \xi^{\dagger}\right\rangle_{\tau}^{q}=\langle\xi \xi\rangle_{\tau}^{q}=\left\langle\xi^{\dagger}\right\rangle_{\tau}^{q}=\langle\xi\rangle_{\tau}^{q}=0,
$$

and

$$
\begin{aligned}
& \left\langle\xi^{\dagger}\left(\mathbf{p}_{T 1}, \mu_{1}, \tau\right) \xi\left(\mathbf{p}_{T 2}, \mu_{2}, \tau\right)\right\rangle_{\tau}^{q} \\
& \quad=\delta\left(\mu_{1}-\mu_{2}\right) \delta^{(2)}\left(\mathbf{p}_{T 1}-\mathbf{p}_{T 2}\right) \frac{1}{e^{\frac{\omega\left(p_{T 1}, \mu_{1}, \tau\right)}{T(\tau)}}-1} .
\end{aligned}
$$

Other expectation values with $\xi$ and $\xi^{\dagger}$ operators can be calculated utilizing Eqs. (57) and (58) and the thermal Wick theorem. To completely specify the quasiparticle representation, one can write $\phi(x)$ and $\partial_{\tau} \phi(x)$ in the following form:

$$
\begin{aligned}
\phi(x)= & \frac{1}{4 \pi} \frac{1}{\sqrt{\pi \tau}} \int_{-\infty}^{+\infty} d \mu d^{2} p_{T} \frac{1}{\sqrt{\omega\left(p_{T}, \mu, \tau\right)}} \\
& \times\left(-i e^{i \mu \eta+i \mathbf{p}_{T} \mathbf{r}_{T}} \xi\left(\mathbf{p}_{T}, \mu, \tau\right)+i e^{-i \mu \eta-i \mathbf{p}_{T} \mathbf{r}_{T}} \xi^{\dagger}\left(\mathbf{p}_{T}, \mu, \tau\right)\right),
\end{aligned}
$$

$$
\begin{aligned}
\partial_{\tau} \phi(x)= & -\frac{1}{4 \pi} \frac{1}{\sqrt{\pi \tau}} \int_{-\infty}^{+\infty} d \mu d^{2} p_{T} \sqrt{\omega\left(p_{T}, \mu, \tau\right)} \\
& \times\left(e^{i \mu \eta+i \mathbf{p}_{T} \mathbf{r}_{T}} \xi\left(\mathbf{p}_{T}, \mu, \tau\right)+e^{-i \mu \eta-i \mathbf{p}_{T} \mathbf{r}_{T}} \xi^{\dagger}\left(\mathbf{p}_{T}, \mu, \tau\right)\right) .
\end{aligned}
$$

In obtaining the above expressions, we have used Eqs. (21), (26), (27), (47), (48), (54), and (55).

\section{MOMENTUM SPECTRA OF CREATED PARTICLES}

In this section, we consider particle production associated with the sudden decoupling of the quasiequilibrium state at some hypersurface $\tau=\tau_{f}$. We utilize the Heisenberg representation to describe evolution of the system at $\tau>\tau_{f}$. In this representation the state is time independent, and because we have disregarded self-interactions of the scalar field we just assume that evolution at $\tau>\tau_{f}$ is governed by the KleinGordon equation. Note that the mean number of produced particles in the model can diverge just because both longitudinal and transverse sizes are assumed to be infinite. Therefore, in order to relate this model to the real world, we assume that the effective transverse size of the expanding system is finite but large enough. We cannot proceed in the same way with the longitudinal dimension, because the boost invariance of the model will be then destroyed. Nevertheless, this difficulty can be circumvented if do not integrate particle momentum spectra over $\theta$ and consider particle momentum spectra in the central rapidity region only.

To obtain meaningful results one needs to define the normalization condition, and such a condition should be consistent with the definition of the $u^{\nu}(x) / T(x)$ field at the hypersurface $\tau=\tau_{f}$ by means of Eq. (1). Perhaps the simplest idea is to assume that the renormalized energymomentum tensor is defined by the subtraction of the expectation value with the corresponding zero-temperature ground state, $T_{\text {ren }}^{\mu \nu}(x)=T^{\mu \nu}(x)-\left\langle 0_{\tau_{f}}\left|T^{\mu \nu}(x)\right| 0_{\tau_{f}}\right\rangle$; see Ref. [29]. Such a subtraction can be understood as the normal ordering with respect to the $\xi^{\dagger}\left(\mathbf{p}_{T}, \mu, \tau_{f}\right)$ and $\xi\left(\mathbf{p}_{T}, \mu, \tau_{f}\right)$ operators. However, while such a renormalization procedure has some attractive features, ${ }^{2}$ it has a drawback from a physical perspective. Namely, the defining property, $\left\langle 0_{\tau_{f}}\left|T_{\text {ren }}^{\mu \nu}(x)\right| 0_{\tau_{f}}\right\rangle=0$, is tailored to the proper time $\tau_{f}$ and to the quasiparticle vacuum $\left|0_{\tau_{f}}\right\rangle$. Then the renormalized energy-momentum tensor does not vanish in the ordinary Minkowski vacuum. Therefore, we define the renormalization procedure by subtracting the expectation value with the Minkowski vacuum,

$$
T_{\text {ren }}^{\mu \nu}(x)=T^{\mu \nu}(x)-\left\langle 0\left|T^{\mu \nu}(x)\right| 0\right\rangle .
$$

We assume that field operators at $\tau=\tau_{f}$ are defined in the quasiparticle representation by Eqs. (59) and (60), and that their further evolution is governed by the KleinGordon equation. To perform a smooth interpolation between the quasiparticle vacuum at $\tau=\tau_{f},\left|0_{\tau_{f}}\right\rangle$, and the ordinary Minkowski vacuum, $|0\rangle$, we take into account that the Bogolyubov coefficient $\beta$ [see Eqs. (40) and (52)] tends to zero when $\tau$ tends to infinity. It allows us to use the quasiparticle creation, $\xi^{\dagger}\left(\mathbf{p}_{T}, \mu, \tau\right)$, and annihilation, $\xi\left(\mathbf{p}_{T}, \mu, \tau\right)$, operators at intermediate proper times $\tau_{f}<\tau<$ $\infty$ to interpolate between quasiparticle and particle degrees of freedom. Note that the instantaneous quasiparticle vacuum, $\left|0_{\tau}\right\rangle$, is situated in the future light cone and is defined as the eigenstate of the normal ordered with respect to the $\xi^{\dagger}\left(\mathbf{p}_{T}, \mu, \tau\right)$ and $\xi\left(\mathbf{p}_{T}, \mu, \tau\right)$ operator $H^{[\tau]}=$ $\int d \sigma u_{\nu}(x) u_{\mu}(x) T^{\mu \nu}(x)$ with the lowest eigenvalue at the three-dimensional hypersurface $\tau=$ const. Since $H^{[\tau]}$ turns out to be $\tau$-dependent, the corresponding instantaneous vacuum state continuously evolves with $\tau$, and approaches the Minkowski vacuum state at asymptotic proper times: $\left|0_{\tau \rightarrow \infty}\right\rangle=|0\rangle$.

It is worth noting that $\xi^{\dagger}\left(\mathbf{p}_{T}, \mu, \tau\right)$ and $\xi\left(\mathbf{p}_{T}, \mu, \tau\right)$ have nontrivial time dependence even while the field satisfies the free evolution equation. It leads to nonconservation of the quasiparticle number and momentum spectra during evolution along the timelike direction $u^{\mu}(x)$. To determine the spectrum of quasiparticles at intermediate proper times, one can utilize Eqs. (47) and (48) and relate $\xi^{\dagger}\left(\mathbf{p}_{T}, \mu, \tau\right)$ and $\xi\left(\mathbf{p}_{T}, \mu, \tau\right)$ with $b^{\dagger}\left(\mathbf{p}_{T}, \mu\right)$ and $b\left(\mathbf{p}_{T}, \mu\right)$, and then utilize Eqs. (44) and (45) to relate $b^{\dagger}\left(\mathbf{p}_{T}, \mu\right)$ and $b\left(\mathbf{p}_{T}, \mu\right)$ with

\footnotetext{
${ }^{2}$ It was shown in Ref. [29] that the expectation value of the energy-momentum tensor of the quasiparticles, $\left\langle T^{\mu \nu}\left(\tau, \mathbf{r}_{T}, \eta\right)\right\rangle_{\tau}^{q}-$ $\left\langle 0_{\tau}\left|T^{\mu \nu}\left(\tau, \mathbf{r}_{T}, \eta\right)\right| 0_{\tau}\right\rangle$, has the perfect fluid form.
} 
$\xi^{\dagger}\left(\mathbf{p}_{T}, \mu, \tau_{f}\right)$ and $\xi\left(\mathbf{p}_{T}, \mu, \tau_{f}\right)$. Then the time-dependent creation, $\xi^{\dagger}\left(\mathbf{p}_{T}, \mu, \tau\right)$, and annihilation, $\xi\left(\mathbf{p}_{T}, \mu, \tau\right)$, operators are related to $\xi\left(\mathbf{p}_{T}, \mu, \tau_{f}\right)$ and $\xi\left(\mathbf{p}_{T}, \mu, \tau_{f}\right)$ operators via the time-dependent Bogolyubov transformation,

$$
\begin{gathered}
\xi\left(\mathbf{p}_{T}, \mu, \tau\right)=u\left(\mathbf{p}_{T}, \mu, \tau\right) \xi\left(\mathbf{p}_{T}, \mu, \tau_{f}\right)+v^{*}\left(\mathbf{p}_{T}, \mu, \tau\right) \xi^{\dagger}\left(-\mathbf{p}_{T},-\mu, \tau_{f}\right), \\
\xi^{\dagger}\left(\mathbf{p}_{T}, \mu, \tau\right)=u^{*}\left(\mathbf{p}_{T}, \mu, \tau\right) \xi^{\dagger}\left(\mathbf{p}_{T}, \mu, \tau_{f}\right)+v\left(\mathbf{p}_{T}, \mu, \tau\right) \xi\left(-\mathbf{p}_{T},-\mu, \tau_{f}\right),
\end{gathered}
$$

where the Bogolyubov coefficients $u\left(\mathbf{p}_{T}, \mu, \tau\right)$ and $v\left(\mathbf{p}_{T}, \mu, \tau\right)$ are

$$
\begin{gathered}
u\left(\mathbf{p}_{T}, \mu, \tau\right)=\alpha^{*}\left(\mathbf{p}_{T}, \mu, \tau\right) \alpha\left(\mathbf{p}_{T}, \mu, \tau_{f}\right)-\beta^{*}\left(\mathbf{p}_{T}, \mu, \tau\right) \beta\left(-\mathbf{p}_{T},-\mu, \tau_{f}\right) \\
v\left(\mathbf{p}_{T}, \mu, \tau\right)=\alpha\left(\mathbf{p}_{T}, \mu, \tau\right) \beta\left(\mathbf{p}_{T}, \mu, \tau_{f}\right)-\beta\left(\mathbf{p}_{T}, \mu, \tau\right) \alpha\left(-\mathbf{p}_{T},-\mu, \tau_{f}\right)
\end{gathered}
$$

Taking into account symmetry $\mathbf{p}_{T} \rightarrow-\mathbf{p}_{T}, \mu \rightarrow-\mu$ properties of the coefficients, it is straightforward to confirm that

$$
\left|u\left(\mathbf{p}_{T}, \mu, \tau\right)\right|^{2}-\left|v\left(\mathbf{p}_{T}, \mu, \tau\right)\right|^{2}=1 .
$$

Equations (62) and (63) mean that the quasiparticle vacuum state at $\tau=\tau_{f}$ is the excited state from the perspective of the quasiparticles at $\tau>\tau_{f}$. It provides that evolution is accompanied by quasiparticle production in an analogy with particle production in an expanding universe [30] (see also Refs. [18,23,31,32].

Taking into account Eqs. (58), (62), and (63), we get that at $\tau>\tau_{f}$

$$
\left\langle\xi^{\dagger}\left(\mathbf{p}_{T 1}, \mu_{1}, \tau\right) \xi\left(\mathbf{p}_{T 2}, \mu_{2}, \tau\right)\right\rangle_{\tau_{f}}^{q}=\delta\left(\mu_{1}-\mu_{2}\right) \delta^{(2)}\left(\mathbf{p}_{T 1}-\mathbf{p}_{T 2}\right)\left(\frac{1+2\left|v\left(\mathbf{p}_{T 1}, \mu_{1}, \tau\right)\right|^{2}}{e^{\frac{\omega\left(p_{T T}, \mu_{1}, \tau_{f}\right)}{T\left(\tau_{f}\right)}}-1}+\left|v\left(\mathbf{p}_{T 1}, \mu_{1}, \tau\right)\right|^{2}\right) .
$$

It is worth noting that $\left\langle\xi^{\dagger}\left(\mathbf{p}_{T}, \mu, \tau\right)\right\rangle_{\tau_{f}}^{q}=\left\langle\xi\left(\mathbf{p}_{T}, \mu, \tau\right)\right\rangle_{\tau_{f}}^{q}=0$, and that $\left\langle\xi^{\dagger}\left(\mathbf{p}_{T 1}, \mu_{1}, \tau\right) \xi^{\dagger}\left(\mathbf{p}_{T 2}, \mu_{2}, \tau\right)\right\rangle_{\tau_{f}}^{q}$ as well as $\left\langle\xi\left(\mathbf{p}_{T 1}, \mu_{1}, \tau\right) \xi\left(\mathbf{p}_{T 2}, \mu_{2}, \tau\right)\right\rangle_{\tau_{f}}^{q}$ are not identically zero at $\tau>\tau_{f}$. To calculate the corresponding one-particle momentum spectra at asymptotic times,

$$
\omega_{p} \frac{d^{3} N}{d^{3} p}=\frac{d^{3} N}{d^{2} p_{T} d \theta}=\omega_{p}\left\langle a^{\dagger}(\mathbf{p}) a(\mathbf{p})\right\rangle_{\tau_{f}}^{q}
$$

one needs to relate plane-wave modes with quasiparticle modes at $\tau=\tau_{f}$. First, taking into account that $\beta$ tends to zero when $\tau$ tends to infinity and using Eqs. (44) and (45) and Eq. (67), we get

$$
\begin{aligned}
\alpha^{*}\left(\mathbf{p}_{T 1}, \mu_{1}, \tau \rightarrow \infty\right) \alpha\left(\mathbf{p}_{T 2}, \mu_{2}, \tau \rightarrow \infty\right)\left\langle\xi^{\dagger}\left(\mathbf{p}_{T 1}, \mu_{1}, \tau \rightarrow \infty\right) \xi\left(\mathbf{p}_{T 2}, \mu_{2}, \tau \rightarrow \infty\right)\right\rangle_{\tau_{f}}^{q} \\
=\left\langle b^{\dagger}\left(\mathbf{p}_{T 1}, \mu_{1}\right) b\left(\mathbf{p}_{T 2}, \mu_{2}\right)\right\rangle_{\tau_{f}}^{q}=\delta\left(\mu_{1}-\mu_{2}\right) \delta^{(2)}\left(\mathbf{p}_{T 1}-\mathbf{p}_{T 2}\right) \\
\quad \times\left(\frac{1+2\left|\beta\left(\mathbf{p}_{T 1}, \mu_{1}, \tau_{f}\right)\right|^{2}}{e^{\frac{\omega\left(p_{T 1}, \mu_{1}, \tau_{f}\right)}{T\left(\tau_{f}\right)}}-1}+\left|\beta\left(\mathbf{p}_{T 1}, \mu_{1}, \tau_{f}\right)\right|^{2}\right)
\end{aligned}
$$

Here we took into account that $\left|\alpha\left(\mathbf{p}_{T}, \mu, \tau \rightarrow \infty\right)\right|=1$. It is worth noting that $\left\langle b^{\dagger}\right\rangle_{\tau_{f}}^{q}=\langle b\rangle_{\tau_{f}}^{q}=0$, and that $\left\langle b^{\dagger} b^{\dagger}\right\rangle_{\tau_{f}}^{q}$ as well as $\langle b b\rangle_{\tau_{f}}^{q}$ are not identically zero. Then, utilization of Eqs. (34) and (35) yields

$$
\left\langle a^{\dagger}\left(\mathbf{p}_{1}\right) a\left(\mathbf{p}_{2}\right)\right\rangle_{\tau_{f}}^{q}=\frac{1}{2 \pi \sqrt{\omega_{p 1} \omega_{p 2}}} \delta^{(2)}\left(\mathbf{p}_{T 1}-\mathbf{p}_{T 2}\right) \int_{-\infty}^{+\infty} d \mu e^{-i \mu\left(\theta_{1}-\theta_{2}\right)}\left(\frac{1+2\left|\beta\left(\mathbf{p}_{T 1}, \mu, \tau_{f}\right)\right|^{2}}{e^{\frac{\omega\left(p_{T T}, \mu_{1}, \tau_{f}\right)}{T\left(\tau_{f}\right)}}-1}+\left|\beta\left(\mathbf{p}_{T 1}, \mu, \tau_{f}\right)\right|^{2}\right) .
$$

Nonthermal contributions into Eqs. (69) and (70) appear due to the difference between the quasiparticle vacuum state and the global Minkowski vacuum state: the zero-temperature ground state $\left|0_{\tau_{f}}\right\rangle$ is an excited state with respect to the observed plane-wave particles. Approximations of the corresponding expressions for $m_{T} \tau_{f} \gg 1$ can be found in Ref. [16]. In a sense, Eq. (70) immediately follows if one relates plane-wave modes with quasiparticle modes at $\tau=\tau_{f}$ by means of Eqs. (34), 
(35), (44), and (45). However, one needs to take into account that Eq. (70) describes the actual particle momentum spectra at asymptotic times only.

Utilizing ultraviolet asymptotic expansions for the Hankel functions, one can show that the expectation value of the renormalized energy-momentum tensor (61), $\left\langle T_{\text {ren }}^{\mu \nu}(x)\right\rangle_{\tau_{f}}^{q}$, diverges. The divergent contributions appear due to the difference between the quasiparticle vacuum state and the global Minkowski vacuum, $\left(\left\langle 0_{\tau_{f}}\left|T^{\mu \nu}(x)\right| 0_{\tau_{f}}\right\rangle-\right.$ $\left.\left\langle 0\left|T^{\mu \nu}(x)\right| 0\right\rangle\right)$, see Ref. [29].

As a final comment we would like to point out that if one neglects the vacuum particle production terms in Eq. (70), then the one-particle momentum spectrum (68) coincides with the spectrum of the local-equilibrium ideal BoseEinstein gas. Indeed, making the substitution $\delta^{(2)}\left(\mathbf{p}_{T 1}-\right.$ $\left.\mathbf{p}_{T 2}\right) \rightarrow(2 \pi)^{-2} R_{T}^{2}$ at $\mathbf{p}_{T 1}=\mathbf{p}_{T 2}$, changing the integration variable $\mu=\left(m_{T} \tau_{f}\right) \sinh (\eta-\theta)$, and calling the integration over the transverse dimension the effective transverse size of the system $R_{T}^{2}$, we obtain

$$
\begin{aligned}
& \frac{R_{T}^{2}}{(2 \pi)^{3}} \int_{-\infty}^{+\infty} d \eta m_{T} \tau_{f} \cosh (\eta-\theta) \frac{1}{e^{\frac{m_{T} \cosh (\eta-\theta)}{T\left(\tau_{f}\right)}}-1} \\
& \quad=\int_{\sigma_{f}} d \sigma_{f} u^{\mu} p_{\mu} \frac{1}{(2 \pi)^{3}} \frac{1}{e^{\frac{u^{\mu} p_{\mu}}{T\left(\tau_{f}\right)}}-1},
\end{aligned}
$$

where $(2 \pi)^{-3}\left(e^{\frac{u^{\mu} p_{\mu}}{T\left(\tau_{f}\right)}}-1\right)^{-1}$ corresponds to the Bose-Einstein local equilibrium distribution function of the ideal gas, $u^{\mu}$ is given by (6), and $p^{\mu}=\left(m_{T} \cosh \theta, \mathbf{p}_{T}, m_{T} \sinh \theta\right)$.

\section{CONCLUSIONS}

Acceptability of hydrodynamics for the description of particle production in the relativistic nucleus and particle collisions means that a true state of the system can be substituted by some proxy state. Such a reduced description can be fulfilled based on the quasiequilibrium statistical operator. In general, the ground state of this statistical operator may not coincide with the Minkowski vacuum. In the present work, we analyze particle production after sudden decoupling of the quasiequilibrium state of the expanding system created in an ultrarelativistic collision. Because the spacetime region occupied by the matter produced in an ultrarelativistic collision lies in the future light cone of a collision, initial conditions and subsequent evolution are described by utilizing some appropriate curved coordinate system in the light cone. To make the problem tractable, we consider a simple but reliable model of the quasiequilibrium state of the noninteracting boostinvariant expanding scalar quantum field. We have performed an exact diagonalization of the model in the quasiparticle representation by means of the Bogolyubov transformation. It allowed us to explicitly disclose the zerotemperature ground state (the instantaneous quasiparticle vacuum). Then, we analyze particle production induced by the sudden decoupling of the quasiequilibrium state of the system. It is noteworthy that the mechanism of particle creation from little bang fireballs created in ultrarelativistic heavy ion and particle collisions has some similarities with the cosmological particle creation.

It is worth noting that contributions to particle momentum spectra from the quasiequilibrium ground state could be observed in relativistic particle and nucleus collisions. Specifically, it was proposed [16] that peculiarities of the measured in $p+p$ collisions at the LHC [33-37] BoseEinstein momentum correlations of two identical charged pions could be attributed to the two-source mechanism of particle emission associated with the decoupling of the quasiequilibrium state. $^{3}$ To make possible quantitative comparison with experimental data, a generalization of the model (accounting for field interactions, expansion in the transverse direction, feed-downs from the resonance decays, etc.) should be performed.

${ }^{3}$ Notice that the existence of two scales in $p+p$ collisions was also proposed in Ref. [38] based on a different underlying physical picture.
[1] Chun Shen, Nucl. Phys. A1005, 121788 (2021).

[2] D. N. Zubarev, A. V. Prozorkevich, and S. A. Smolyanskii, TMF 40, 394 (1979); Theor. Math. Phys. 40, 821 (1979).

[3] A. Hosoya, M.-a. Sakagami, and M. Takao, Ann. Phys. (N.Y.) 154, 229 (1984).

[4] D. Zubarev, V. Morozov, and G. Röpke, Statistical Mechanics of Nonequilibrium Processes. Volume 1: Basic Concepts. Kinetic Theory (Berlin, Akademie Verlag, 1996).

[5] D. Zubarev, V. Morozov, and G. Röpke, Statistical Mechanics of Nonequilibrium Processes. Volume 2:
Relaxation and Hydrodynamic Processes (Berlin, Akademie Verlag, 1997).

[6] F. Becattini, L. Bucciantini, E. Grossi, and L. Tinti, Eur. Phys. J. C 75, 191 (2015).

[7] T. Hayata, Y. Hidaka, T. Noumi, and M. Hongo, Phys. Rev. D 92, 065008 (2015).

[8] A. Harutyunyan, A. Sedrakian, and D. H. Rischke, Particles 1, 155 (2018).

[9] F. Becattini, M. Buzzegoli, and E. Grossi, Particles 2, 197 (2019). 
[10] D. Blaschke, G. Röpke, D. N. Voskresensky, and V. G. Morozov, Particles 3, 380 (2020).

[11] E. T. Jaynes, Phys. Rev. 106, 620 (1957).

[12] E. T. Jaynes, Phys. Rev. 108, 171 (1957).

[13] Yu. M. Sinyukov, Preprint ITP-93-8E, 1993.

[14] Yu. M. Sinyukov, Nucl. Phys. A566, 589c (1994).

[15] Yu. M. Sinyukov, Heavy Ion Phys. 10, 113 (1999).

[16] S. V. Akkelin, Eur. Phys. J. A 55, 78 (2019).

[17] J. D. Bjorken, Phys. Rev. D 27, 140 (1983).

[18] N. D. Birrell and P.C. W. Davies, Quantum Fields in Curved Space (Cambridge University Press, Cambridge, England, 1984).

[19] C. M. Sommerfield, Ann. Phys. (N.Y.) 84, 285 (1974).

[20] N. B. Narozhny, A. M. Fedotov, B. M. Karnakov, V. D. Mur, and V. A. Belinskii, Phys. Rev. D 65, 025004 (2001).

[21] L. C. B. Crispino, A. Higuchi, and G. E. A. Matsas, Rev. Mod. Phys. 80, 787 (2008).

[22] I. S. Gradshteyn and I. M. Ryzhik, Table of Integrals, Series and Products (Academic, New York, 1980).

[23] A. A. Grib, S. G. Mamayev, and V. M. Mostepanenko, Vacuum Quantum Effects in Strong Fields (Friedmann Laboratory Publishing, St. Petersburg, 1994).

[24] L. Mandel and E. Wolf, Optical Coherence and Quantum Optics (Cambridge University Press, Cambridge, England, 1995).

[25] J.-P. Gazeau, Coherent States in Quantum Physics (WileyVCH Verlag Gmbh Co. KGaA, Weinheim, 2009).
[26] C. Bloch and C. De Dominicis, Nucl. Phys. 7, 459 (1958).

[27] N. N. Bogolubov and N. N. Bogolubov, Jr., An Introduction to Quantum Statistical Mechanics (Gordon and Breach, New York, 1992).

[28] S. R. de Groot, W. A. van Leeuwen, and Ch. G. van Weert, Relativistic Kinetic Theory (North-Holland, Amsterdam, 1980).

[29] D. Rindori, L. Tinti, F. Becattini, and D. Rischke, arXiv: 2102.09016.

[30] L. Parker, Phys. Rev. 183, 1057 (1969).

[31] S. A. Fulling, Aspects of Quantum Field Theory in Curved Space-Time (Cambridge University Press, Cambridge, England, 1989).

[32] L. E. Parker and D. J. Toms, Quantum Field Theory in Curved Spacetime (Cambridge University Press, Cambridge, England, 2009).

[33] K. Aamodt et al. (ALICE Collaboration), Phys. Rev. D 84, 112004 (2011).

[34] S. Acharya et al. (ALICE Collaboration), J. High Energy Phys. 09 (2019) 108.

[35] ATLAS Collaboration, Eur. Phys. J. C 75, 466 (2015).

[36] A. M. Sirunyan et al. (CMS Collaboration), Phys. Rev. C 97, 064912 (2018).

[37] A. M. Sirunyan et al. (CMS Collaboration), J. High Energy Phys. 03 (2020) 014.

[38] V. A. Khoze, A. D. Martin, M. G. Ryskin, and V. A. Schegelsky, Eur. Phys. J. C 76, 193 (2016). 